\title{
Defining the Mechanism of Action of Herbal Therapies in Rheumatoid Arthritis: Is This the Road to Clinical Development and Acceptance?
}

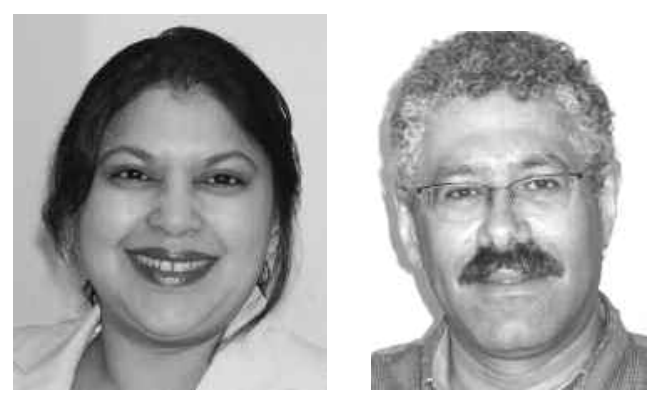

Herbal treatments are widely used to treat chronic inflammatory disorders such as rheumatoid arthritis (RA). This is particularly the case in China, where these treatments have been part of traditional medicine practice, in some cases for centuries. In the current age of targeted therapies for RA, where efficacy, safety, and mechanism of action are demonstrated using widely accepted scientific methodology, it remains challenging to determine how the spectrum of herbal and other traditional therapies should fit into modern therapeutic paradigms. Patients not uncommonly gravitate toward these treatments because of their "natural" origins, their perceived safety, and their relatively modest cost. Yet in most cases, evidence for clinical efficacy, safety, and mode of action is lacking, leading many clinicians to advise their patients against the use of such treatments until better evidence is available. A comprehensive analysis of the available clinical evidence for a spectrum of herbal therapies used in the treatment of RA has recently been updated in the Cochrane Library ${ }^{1}$. This database is a valuable resource in helping clinicians begin to navigate through the confusing and often contradictory information that is widely available regarding use of these therapies and their influence on disease. In most cases, even less is known about how any individual agent potentially affects the complex molecular pathways underlying the clinical phenomenology seen in RA.

In this issue of The Journal, Hsu, et al begin this challenging task in relation to Plectranthus amboinicus, a herbal therapy widely used in Taiwan to treat RA and other inflammatory disorders ${ }^{2}$.

One of the best-studied herbal treatments in RA to date has been thunder god vine (Tripterygium wilfordii hook F, TwHF). Several randomized clinical trials have demonstrated efficacy ${ }^{3,4,5}$, with the most recent showing benefits that were at least as potent as sulfasalazine ${ }^{3}$. Unfortunately, the studies demonstrated a number of serious toxicities such as aplastic anemia, which has dampened the enthusiasm for recommending widespread use of the treatment. Topical versions of thunder god vine have also demonstrated efficacy in controlled trials, with less toxicity than was observed with the systemic administration ${ }^{6}$.

Because of the encouraging clinical efficacy of TwHF, considerable investigative effort was put into defining the active ingredient(s). Largely through the tenacious efforts of Dr. Peter Lipsky and his colleagues, several ingredients were found to have antiinflammatory and immunosuppressive effects, primarily through the suppression of proinflammatory gene expression. The most potent and abundant of these compounds was triptolide, which was subsequently shown to have impressive benefits in an animal model of systemic lupus erythematosus ${ }^{7}$.

The contribution of $\mathrm{Hsu}$, et al in this issue ${ }^{2}$ demonstrates that rosmarinic acid (RosA), the active ingredient in the herbal treatment $P$. amboinicus, has significant effects on articular damage in an animal model of RA, mediated through its effects on osteoclastogenesis. The authors had previously demonstrated antiinflammatory effects in the same model mediated through inhibition of proinflammatory cytokines such as interleukin 6 (IL-6), tumor necrosis factor- $\alpha$ (TNF- $\alpha)$, and IL- $1 \beta^{8}$. The effects on osteoclastogenesis suggest that this treatment may have anti-erosive effects in inflammatory arthritis.

Bone remodeling involves synthesis of bone matrix by osteoblasts and resorption of bone by osteoclasts. Dysregulation of this mechanism can be affected by several factors that include immune and inflammatory mediators. The link between immune regulation and bone homeostasis has been well established in recent years. Consequently, the area of research focused on understanding the crosstalk between the immune and the skeletal system is now termed "osteoimmunology" . Two chronic inflammatory diseases that result in associated bone loss are RA and periodontal disease, which has prompted research into understanding

See Plectranthus amboinicus inhibits osteoclastogenesis, page 1844 
the link between immune cells and bone remodeling. Key molecules of signaling pathways in osteoimmunology are the receptor activator of nuclear factor- $\mathrm{\kappa B}$ (RANK), its ligand RANKL, and osteoprotegrin (OPG), which is a physiological inhibitor of the RANK/RANKL interaction. These molecules are key factors that regulate bone remodeling, and this system is modulated by various inflammatory cytokines and immune mediators, primarily by influencing the RANKL-OPG expression ratio ${ }^{10,11}$.

RANK, a member of the TNFR superfamily (TNFRSF11A), is activated on hematopoietic progenitors, which further differentiate into multinucleated osteoclasts ${ }^{10}$. RANK is also expressed on innate immune cells such as dendritic cells ${ }^{12}$. The signaling ligand for RANK is RANKL (TNFSF11), a type II transmembrane protein belonging to the TNF superfamily. RANKL is expressed in bone mainly by mesenchymal cells such as osteoblasts and stromal cells $^{11}$, as well as on immune cells such as lymphocytes ${ }^{13}$. Activation of RANK by RANKL in osteoclast precursor cells mediates the activation of several downstream signaling pathways that includes activation of the key inflammatory transcription factor nuclear factor- B, which in turn promotes osteoclast maturation ${ }^{14}$. Interestingly, several immune mediators such as TNF- $\alpha$, IL-1 13 , IL- 6 , IL-17, and vitamin D induce the expression of RANKL and affect osteoclast activity by altering RANK signaling ${ }^{11,15}$. Consistent with this, it has been shown that activated $\mathrm{T}$ cells expressing RANKL can also mediate bone loss and joint destruction ${ }^{16}$. In vivo studies have shown that genetic mutations in either RANK or RANKL influence both osteoclast and lymph node development ${ }^{17}$.

Despite all the inferred evidence, the regulation of RANK/RANKL system by immune/inflammatory mediators and its effects on bone remodeling are not completely elucidated at the molecular level. However, the RANK-RANKL axis is now well established as a therapeutic target for diseases such as osteoporosis, RA, and bone tumors ${ }^{18,19}$. For example, a monoclonal antibody (denosumab) targeting RANKL shows efficacy in osteoporosis and bone metastases ${ }^{20}$.

$\mathrm{Hsu}$, et $a l^{2}$ demonstrate that RosA affects osteoclastogenesis by altering the RANKL-mediated signaling pathways. They demonstrated that RANKL-induced tartrate-resistant acid phosphatase (TRAP), a marker for osteoclast differentiation, was decreased in activated macrophages by RosA, without significantly affecting cell viability. They showed that RosA inhibited RANKL-mediated induction of $c$-fos, DC-STAMP, NFATcl, and integrin $\beta 3$ gene expression, all cell markers of osteoclast differentiation, in activated macrophages. They also showed that RANKL-induced activation of p38, ERK, and nuclear factor- $\mathrm{KB}$, as well as nuclear translocation of NFATc1, was significantly inhibited by RosA. As osteoclasts are derived from macrophage/monocyte precursors, the authors investi- gated the effect of RosA on RANKL-mediated responses in macrophages stimulated with macrophage colony-stimulating factors (M-CSF). It should be noted that cultivation of macrophages with M-CSF and RANKL serves to provide multinucleated osteoclasts with increased expression of the dendritic cell-specific marker DC-STAMP, which has been demonstrated to be essential for the formation of multinuclear giant cells in osteoclastogenesis ${ }^{21}$. This is consistent with previous studies that have indicated that RANK/RANKL interactions are not only important for understanding the mechanism of bone loss, but in addition these interactions regulate several immune functions that include dendritic cell/T cell crosstalk, dendritic cell survival, and lymph node development ${ }^{13}$. Therefore it would be important to elucidate, at the molecular level, the effects of the phytochemical RosA on dendritic cell and $\mathrm{T}$ cell crosstalk during inflammation, and on lymphocyte polarization and development.

As phytochemical RosA is extracted from the medicinal plant $P$. amboinicus, in this study the authors further investigated the effect of the native plant in a murine model of collagen-induced arthritis (CIA). They showed that the arthritic score and paw thickness was reduced after 6 weeks of immunization, while the serum levels of the proinflammatory cytokines TNF- $\alpha$ and IL-1 $1 \beta$ were significantly suppressed by day 50 in this model. Although mechanistically appealing, a common and fundamental element of the molecular pathogenesis of immune-mediated chronic inflammatory diseases is that there is a broad dysregulation of cytokine and chemokine networks, rather than simply changes in the serum level of one or 2 cytokines. Thus, the effect of the herbal treatment on the production of a wide range of pro- and antiinflammatory cytokines such as IL-4, IL-6, IL-10, IL-15, IL-18, IL-23, IL-17, and IL-32, chemokines such as monocyte chemoattractant protein-1 and matrix-degrading matrix metalloproteinases should be clarified in the animal model, both at the systemic and articular tissue levels.

This work provides important proof-of-principle information that this Taiwanese medicinal plant may have antiinflammatory and anti-erosive properties that could potentially be beneficial in the treatment of destructive inflammatory arthropathies such as RA. The data demonstrate that key signaling pathways are modulated by this herbal treatment. Importantly, it is clearly shown that RosA is the active ingredient responsible for this, since the dosimetry of a single compound is more reliably calculated, administered, and tested than the parent herbal preparation P. amboinicus.

Is this enough to move ahead with the needed human studies that would potentially demonstrate efficacy and safety of $P$. amboinicus in RA? The fact that the herbal treatment is widely used in traditional Taiwanese medicinal practice may be somewhat reassuring with regard to the latter. Unfortunately, clearly demonstrating clinical efficacy in RA Personal non-commercial use only. The Journal of Rheumatology Copyright @ 2011 . All rights reserved. 
has become a very expensive undertaking, requiring a substantial financial commitment and extensive clinical trial infrastructure. In a marketplace where there are multiple, highly effective biologic therapies and a rapidly declining eligible study patient population, the demonstration of clinical efficacy of a herbal compound such as $P$. amboinicus becomes a truly daunting task.

\section{NEELOFFER MOOKHERJEE, $\mathrm{PhD}$; HANI EL-GABALAWY, MD,} Manitoba Centre for Proteomics and Systems Biology, Department of Internal Medicine, University of Manitoba, Winnipeg, MB, Canada

Address correspondence to Dr. El-Gabalawy.

E-mail:elgabalh@cc.umanitoba.ca

\section{REFERENCES}

1. Cameron M, Gagnier JJ, Chrubasik S. Herbal therapy for treating rheumatoid arthritis. Cochrane Database Syst Rev 2011;CD002948.

2. Hsu Y-C, Cheng C-P, Chang D-M. Plectranthus amboinicus attenuates inflammatory bone erosion in mice with collagen-induced arthritis by downregulation of RANKL-induced NFATc1 expression. J Rheumatol 2011;38:1844-57.

3. Goldbach-Mansky R, Wilson M, Fleischmann R, Olsen N, Silverfield J, Kempf P, et al. Comparison of Tripterygium wilfordii Hook $\mathrm{F}$ versus sulfasalazine in the treatment of rheumatoid arthritis: a randomized trial. Ann Intern Med 2009;151:229-40, W49-51.

4. Tao X, Younger J, Fan FZ, Wang B, Lipsky PE. Benefit of an extract of Tripterygium wilfordii Hook F in patients with rheumatoid arthritis: a double-blind, placebo-controlled study. Arthritis Rheum 2002;46:1735-43.

5. Tao XL, Sun Y, Dong Y, Xiao YL, Hu DW, Shi YP, et al. A prospective, controlled, double-blind, cross-over study of tripterygium wilfordii hook $\mathrm{F}$ in treatment of rheumatoid arthritis. Chin Med J (Engl) 1989;102:327-32.

6. Cibere J, Deng Z, Lin Y, Ou R, He Y, Wang Z, et al. A randomized double blind, placebo controlled trial of topical Tripterygium wilfordii in rheumatoid arthritis: reanalysis using logistic regression analysis. J Rheumatol 2003;30:465-7.
7. Tao X, Fan F, Hoffmann V, Longo NS, Lipsky PE. Therapeutic impact of the ethyl acetate extract of Tripterygium wilfordii Hook F on nephritis in NZB/W F1 mice. Arthritis Res Ther 2006;8:R24.

8. Chang JM, Cheng CM, Hung LM, Chung YS, Wu RY. Potential use of Plectranthus amboinicus in the treatment of rheumatoid arthritis. Evid Based Complement Alternat Med 2010;7:115-20.

9. Arron JR, Choi Y. Bone versus immune system. Nature 2000;408:535-6.

10. Gruber R. Cell biology of osteoimmunology. Wien Med Wochenschr 2010;160:438-45.

11. Hanada R, Hanada T, Penninger JM. Physiology and pathophysiology of the RANKL/RANK system. Biol Chem 2010;391:1365-70.

12. So T, Lee SW, Croft M. Tumor necrosis factor/tumor necrosis factor receptor family members that positively regulate immunity. Int J Hematol 2006;83:1-11

13. Theill LE, Boyle WJ, Penninger JM. RANK-L and RANK: T cells, bone loss, and mammalian evolution. Annu Rev Immunol 2002;20:795-823

14. Riches PL, Ralston SH. Recent insights into the biology of bone turnover. J R Coll Physicians Edinb 2010;40:66-9.

15. Leibbrandt A, Penninger JM. RANK/RANKL: regulators of immune responses and bone physiology. Ann NY Acad Sci 2008;1143:123-50.

16. Horwood N. Lymphocyte-derived cytokines in inflammatory arthritis. Autoimmunity 2008;41:230-8.

17. Kobayashi Y, Udagawa N, Takahashi N. Action of RANKL and OPG for osteoclastogenesis. Crit Rev Eukaryot Gene Expr 2009;19:61-72.

18. Nakashima T, Wada T, Penninger JM. RANKL and RANK as novel therapeutic targets for arthritis. Curr Opin Rheumatol 2003; 15:280-7.

19. Wittrant Y, Theoleyre S, Chipoy C, Padrines M, Blanchard F, Heymann D, et al. RANKL/RANK/OPG: new therapeutic targets in bone tumours and associated osteolysis. Biochim Biophys Acta 2004;1704:49-57.

20. Castellano D, Sepulveda JM, Garcia-Escobar I, Rodriguez-Antolin A, Sundlov A, Cortes-Funes H. The role of RANK-ligand inhibition in cancer: the story of denosumab. Oncologist 2011;16:136-45.

21. Yagi M, Miyamoto T, Toyama Y, Suda T. Role of DC-STAMP in cellular fusion of osteoclasts and macrophage giant cells. J Bone Miner Metab 2006;24:355-8.

J Rheumatol 2011;38:1817-19; doi:10.3899/jrheum.110823 